Neurosurg Focus 9 (4):E14, 2000

\title{
Artificial disc technology
}

\author{
Qi-Bin Bao, Ph.D., and Hansen A. Yuan, M.D. \\ Department of Orthopedic Surgery, State University of New York Health Science Center at Syracuse, \\ Syracuse, New York
}

\begin{abstract}
After approximately 40 years of research and development, artificial disc technology may finally be coming of age. A number of devices are either at the late stage of preclinical study or in the early stage of clinical trial, and the results are promising so far. Due to the multicomponent structure of the disc, surgeons performing disc arthroplasty have the option of replacing either the entire disc or a portion of it. The decision will be largely dependent on the pathological entity addressed, the condition of the patient's spinal disc and surrounding tissues, and the cost and potential risk of the procedure. Driven by demand, almost all the emphasis in artificial disc development has been placed on the lumbar disc, with a smaller effort directed toward the cervical disc. No attempt has been made to develop an artificial thoracic disc. However, by examining the differences and similarities in structure, anatomy, function, mechanism of degeneration, pathology, surgical technique, and complications between the lumbar and thoracic disc, the authors believe it is feasible to apply artificial disc technology in the treatment of thoracic disc disease. Nonetheless, due to the rarity of thoracic disc disease and the more stable structure of this spinal component, the demand for artificial disc or artificial nucleus technology for the thoracic disc probably will be smaller than that for lumbar disc technology.
\end{abstract}

KEY WORDS - thoracic disc • artificial disc • artificial nucleus $・$ disc degenerative disease

After some 40 years of research and development, artificial disc technology has finally reached the point at which several designs are either near the end stage of preclinical study or in the early phase of clinical trial, with promising results so far. Although it may still be too early to claim that this technology has matured, it can at least be said to have passed its infancy and even attained its preadolescence. However, in comparison with other artificial joint technologies such as those for the knee and hip, progress in artificial disc technology has been fairly slow.

Is the slow progress of artificial disc development due to the lack of a driving force, namely, lack of need for such a device? The answer is definitely not. Statistics substantiate this statement. Among patients seeking medical treatment for spinal disorders, low-back pain is a common cause. In the United States alone, almost 700,000 spine procedures are performed each year, and the total cost incurred by back pain and its treatment exceeds $\$ 50$ billion per year. Most important, the two most common spinal surgery procedures, discectomy and fusion, are far from ideal for treating spinal disc degenerative disease. Although discectomy has a reasonably good short-term effect in relieving radicular pain, it causes disc height reduction in almost all patients ${ }^{18}$ and further increases the instability of the treated disc. ${ }^{16}$ The consequences of these changes in anatomy and structural stability are twofold.

Abbreviations used in this paper: VATS = video-assisted thoracic surgery.
First, the procedure achieves poor results in relieving back pain. Second, it leads to many secondary complications, such as spinal stenosis and facet pain. As for fusion, by abolishing motion it successfully relieves back pain; however, it also significantly alters the normal biomechanical properties of the intervertebral disc. Although single-level disc fusion may not impair the patient's normal function and activity as seriously as knee or hip fusion, it definitely increases stress and strain on the discs adjacent to the fused disc. ${ }^{24}$ Therefore, the need for more effective treatments for low-back pain is great from both a scientific and an economic point of view.

Scientifically, both physicians and industrial and academic researchers have been seeking better solutions for low-back pain treatment. Naturally, disc arthroplasty has been considered to be the "holy grail" of back pain treatment. The evolution of artificial disc technology is attested in part by experience in numerous patents as reported in publications over the past 40 years, especially the last 10 years.

One of the major reasons why artificial disc technology has been slow to develop is the structural and functional complexity of the disc. Unlike hip and knee joints, which are composed mainly of a layer of cartilage tissue on each articulating surface, the intervertebral disc is composed of three distinctively different tissues: annulus, nucleus, and endplate. The annulus has a multilayered structure, much like that of an automobile tire, with each layer formed from well-organized collagen fibers running in opposite 
directions in any adjacent layers at approximately $30^{\circ}$ to the horizontal plane. This structure provides the annulus with a high tensile modulus and strength, as well as equal torsional modulus in either direction. The nucleus is enclosed within the annulus and the endplates and is composed mainly of a very hydrophilic polymer called glycosaminoglycan, which is capable of absorbing a large amount of water and which forms a gellike matrix. The amount of water absorbed by the nucleus depends not only on the composition of the polymer matrix but also on the external pressure exerted on the disc. At a young age and when the disc is healthy, $80 \%$ of the nucleus is constituted by water, even under normal loading conditions, and this keeps the annulus well inflated. Again, somewhat like an automobile tire, when the annulus is inflated the disc becomes very stiff and stable. Although the cartilage endplate contributes little to the overall mechanical properties of the disc, it plays an important role in allowing nutrients to pass into the disc. It should be noted that the mechanical properties of a disc are a function of the structure and integrity of both annulus and nucleus in combination. A change in either one of these parameters can affect the overall properties of the disc.

Functionally, the intervertebral disc performs two important, but somewhat conflicting, duties: it maintains spinal column stability while providing the column with necessary flexibility. Without intervertebral discs, the human spine is unable to bend and its function is greatly impaired. However, because numerous nerves course alongside the spinal column as well as directly down the spinal cord, the disc has to keep the vertebral bodies separated so as to hold the foramen space open and provide adequate stability.

Disc degeneration often occurs in patients at a much younger age than hip and knee degeneration. Disc degeneration, which is believed to be the major cause of lowback pain, often begins with a structural change in which the nucleus loses its water-binding capacity and the disc consequently deflates. After this happens, more compressive loading shifts to the annulus, rendering this structure more susceptible to delamination and damage. Damage to the annulus, in turn, accelerates the disc degeneration process. Disc degeneration can also accelerate the degeneration of other surrounding tissues, such as facet joints. This degeneration cascade in the disc joint has been well documented. 48

Currently the two most common surgical procedures, discectomy and fusion, at best only address the symptom of low-back pain. Biologically and biomechanically, both procedures actually worsen the condition of the affected disc, adjacent discs, and surrounding tissues, leading to further degeneration. Hence the long-term results of both these procedures are relatively poor. Logically, a better solution is implantation of an artificial disc, which is intended not only to treat low-back pain but also to restore or maintain the normal anatomy and function of the diseased disc.

Several review articles on various types of artificial disc have been published. ${ }^{5,7,26,46}$ Somewhat like hemiarthroplasty in other joints involved, in intervertebral disc replacement one can replace either the entire disc or only its nucleus. The former prosthesis is called an artificial total disc and the latter an artificial nucleus.

\section{Artificial Total Disc}

The artificial total disc is designed to replace the entire disc: annulus, nucleus, and (very often) endplates. Because the function of the endplate is more biological than biomechanical, it often does not need to be preserved after the disc is replaced with nonbiological material(s), unless the design entails articulating the artificial disc surface with the endplate for reduced friction and wear. Most artificial disc designs require removal of the endplates and fixation of the superior and inferior surfaces of the implant to the vertebral bodies. The main benefit of replacing the entire disc is that the disc is consequently less dependent on the integrity of the annulus and the stage of degeneration. Conceptually, artificial discs can be used in patients with disc degeneration at any stage of progression. Because of the added cost and risk involved in implanting such a device, however, in practice its use can often be justified only in patients with more severe disc degeneration.

Due to the complexity of the structure and function of the disc, it has proven difficult to design an artificial total disc that mimics all the mechanical properties of a natural disc while retaining the required durability. A multicomponent design incorporating several materials with very different mechanical properties or a composite structure is often required. In a multicomponent design, researchers are also often faced with problems such as interfacial bonding and wear.

For flexibility, either the material must be elastic itself or the design must have elastic characteristics at least in one direction (preferably in multiple directions). On the other hand, because the implant must maintain a firm fixation to the vertebrae, a hard material such as metal must often be used for the superior and inferior surfaces of the device. Fixation is often achieved by one or a combination of the following mechanisms: 1) anchoring through one or several pegs or posts inserted into the vertebrae; 2) physical interfacing via a threaded surface; 3) promotion of bone ingrowth by means of a porous surface; or 4) fixation with screws through a side wing extending from the plate.

To minimize contact stress, researchers tend to design the device to cover the entire cross-sectional area of the vertebra so that the load can be spread over a large surface area. Although this makes mechanical sense, it renders surgical insertion more difficult and requires almost every artificial disc to be implanted via an open anterior approach with a large incision, and also entails prolonged operative time. Use of a hard or rigid material for superior and inferior surfaces further increases the bulkiness of the implant. Given the current trend of using less or minimal invasive surgery (even for some fusion surgeries), this type of device may have difficulty gaining wide acceptance among surgeons.

In cases of fixation failure, the bulkiness of the artificial total disc could prove catastrophic. Although artificial disc implantation offers some benefits over fusion, it would be difficult to convince surgeons to use the device if the potential risk to patients is higher than that associated with fusion. In view of the fact that the current fusion rate is higher than $90 \%$ for the fusion cage (possibly even higher when using some forms of bone growth factor), 
and given the relatively low incidence of cage migration, the fusion procedure has set a high standard for the artificial disc to meet.

Although the concept of a disc prosthesis was first set forth in 1956 in a French patent by van Steenbrugghe ${ }^{45}$ it was not until 17 years later that Urbaniak, et al. ${ }^{44}$ reported the first disc prosthesis, which was prototyped and implanted into a chimpanzees. Since then, many other disc prosthesis concepts have been proposed, mostly in the patent literature.

Basic design concepts and component material(s) can be divided into three groups: metal, nonmetal, and metal in combination with nonmetal. The main advantage of a disc prosthesis design using metal alone is its inherently high fatigue strength compared with a nonmetal design. Because patients with back pain are on average approximately 40 years old - that is, much younger than the population of patients requiring total hip or total knee patients-it is proposed that the device should have at least 40 years of fatigue life. Researchers who use exclusively metal materials designs believe that they are thereby more effectively addressing the fatigue issue. However, because most metals are much stiffer than the natural disc they are used to replace, they must be designed in special forms to reduce this stiffness and provide needed flexibility.

The most widely tested all-metal disc prosthesis, developed by Kostuik and associates, ${ }^{19,20}$ features two Ti-6Al$4 \mathrm{~V}$ springs pocked between two forged or hot isostatically pressed cobalt-chromium-molybdinum alloy plates with a posterior hinge allowing flexion and extension. In designing the implant an effort was made to meet various design criteria such as biocompatibility, endurance, kinematics, dynamics, and bone fixation. The hinge provides the implant with a full range $\left(15-20^{\circ}\right)$ of axial rotation in the sagittal plane as well as a small amount of rolling lateral rotation $\left(3-6^{\circ}\right)$ to match the physiological range. The springs were chosen to duplicate the stiffness of a natural disc. Vertically projecting lugs are positioned at the front and side of the plate members, through which screws can be placed for fixation. Fatigue tests were conducted on the individual components (100 million cycles), and no failure was found in a total of 17 springs. In a sheep model, it was shown that fibrous tissue does not grow between the hinges or around the coils, at least in the short term. Such ingrowth would be expected to interfere significantly with implant mechanics. However, due to the bulkiness of the device and the potential risk associated with its use, it has yet to be applied in humans.

Whereas the principle advantage of a metal disc prosthesis is its fatigue strength, the primary benefit of a nonmetal design is its mechanical similarity to the natural disc. With its lower elasticity modulus, it more closely replicates disc kinematics. The challenge, however, arises when attempting to develop a long-lasting device (longevity of $\geq 40$ years) and an interface that promotes ingrowth of the bone implant.

The concept of a disc prosthesis was introduced with a nonmetal design by van Steenbrugghe. ${ }^{45}$ The implant comprises a multicomponent disc encompassing intermediate cushion inlayers with a plastic body of varying shapes. However, the inventor did not mention how the implant was to stay in place. No laboratory test has been reported on this device. The most widely tested device in the nonmetal category is that designed by Lee and colleagues. ${ }^{25,31}$ This design features a soft elastomer central core to mimic the function of the natural disc nucleus and reinforcing fiber sheets with specific alternating fiber orientation in six to 15 laminae embedded in a second elastomer to mimic the function of the annulus, as well as two stiff plates. By selecting appropriate materials, the device is able to mimic both the compressive modulus and the compressive-torsional stiffness of the natural disc. ${ }^{23}$ Data on compression-flexion stiffness and compression-extension have not been reported. Lack of adequate implant and vertebra fixation is believed to be the major obstacle to the clinical use of this device.

To take advantage of the benefits of both metal and nonmetal materials and overcome the drawbacks involved in using either of them alone, many researchers have combined both types of materials in their designs. Most commonly this has taken the form of a metal-polymer-metal sandwich design. A metal plate is used to improve fixation by means of spikes, tabs with screws, or porous coating for ingrowth. With the component thus stable and fixed, the polymer may provide the needed flexibility.

Although many artificial disc designs have been proposed over the years, only three have been tested extensively and used clinically. The LINK SB Charité device has undergone the largest and longest clinical trial of any existing artificial disc. It was developed by Buttner-Janz and colleagues ${ }^{8,9}$ in the mid-to-late 1980s. The prosthesis has undergone several major design, structural, and manufacturing modifications from the first to the third and current generation. ${ }^{49}$ The design features two concave endplates composed of a cobalt-chromium-molybdenum alloy. The plates have spikes or teeth that enable them to be fixed without cement to the vertebral body. A biconvex oval polyethylene spacing piece with contours to match the endplates is placed between them. Similar to the balland-socket design of hip joint prosthesis, this device allows rotation in all three directions (unpublished data). However, due to the lack of elasticity of the material, this device cannot replicate the normal compressive stiffness of the natural disc.

Since 1984, well over 2000 prostheses of all three generations have been implanted in Europe. The most detailed clinical data for this device were reported by Griffith, et al., ${ }^{17}$ after a multicenter retrospective study conducted on 93 patients in whom a total of 139 third-generation prostheses were implanted. The predominant surgical indication was for degenerative disc disease, usually occurring at L4-5 or L5-S1. The average length of follow-up review was 11.9 months (range $1-37$ months). Using a 10-point analog pain scale, the data indicated a statistically significant alleviation of pain in both leg and back after disc implantation, with greater gains in relieving leg pain than back pain. The authors stated that the improvements in leg pain might be a result of the degree of decompression achieved by the surgeon and not necessarily a direct effect of the use of prosthesis placement.

Parallel to European developments, Steffee ${ }^{42}$ conducted his pilot clinical study in six patients in the United States between 1988 and 1989 after some design work and laboratory testing. This disc consists of a hexene-based, carbon black-filled polyolefin rubber core vulcanized to two 
titanium plates. Fixation is accomplished with a porous coating to promote ingrowth and four 7-mm conical posts that are socketed into the vertebral body. In the six patients in the preliminary clinical series (as reported in Fernstrom $^{11}$ and in an unpublished study by McMilland and Steffee) there were two prosthesis failures caused by fracture of the rubber core. Several reasons could be evinced to explain this high rate of device failure. First, implants were used at disc levels adjacent to the fusion site in four of the six patients; this may have led to the exertion of severe stress at the implant level. Second, one of the patients had degenerative scoliosis; without secure plate fixation and resistance to intense shear force, deformity should be a contraindication for implanting an artificial total disc. Third, the shear-fatigue strength of this rubber core might not have been sufficient.

More recently, a cap-cup matching articulation device was designed by Marnay. ${ }^{28}$ The implant (ProDisc) is composed of titanium endplates with titanium plasma spray coating on the outer surfaces and a polyethylene core. Marnay (unpublished data) recently reported his clinical results in 50 patients after a minimum 7 years' follow up, with excellent or good results in $78 \%$ of these patients.

\section{Artificial Nucleus}

The nucleus prosthesis approach has several obvious advantages over the total disc prosthesis. By replacing only the nucleus, it preserves the remaining disc tissuesannulus and endplates - and therefore preserves their functions. Because the nucleus has a much simpler structure and function than the annulus and endplates, the design of the nucleus prosthesis allows surgeons to leave the annulus and endplates intact, making the surgical procedure much easier than that required to replace the entire natural disc.

The primary objectives of the nucleus prosthesis are to reinflate the annulus and to relieve the compressive load on this disc component by sharing a significant portion of that load. Another major advantage of the nucleus prosthesis is that it can be implanted by means of a minimally invasive surgical procedure. Depending on the design and choice of material, it is even feasible to implant the device using an endoscopic technique with only a small incision in the annulus. Because the implant is not designed to be affixed to the vertebrae, no fixation component is required. The surgical time required should be comparable to that required for a discectomy.

The risk associated with placement of a nucleus prosthesis should be lower than that associated with a total disc prosthesis. Although implant extrusion remains a primary concern in the design and use of a nucleus implant, it is less likely than a total disc prosthesis to cause permanent injury to the nerve because of its relatively small size and soft consistency. In case of prosthesis failure, it is relatively easy to remove the implant and convert to a primary fusion or perform a disc replacement procedure.

The major limitation of the nucleus prosthesis is that it can be used only in patients in whom disc degeneration is at an early or intermediate stage because it requires the presence of a competent natural annulus. In a disc at a late stage of degeneration, with severe annular tears and delamination, the annulus may not be strong enough to provide the needed constraint. Most nucleus prostheses would also not be suitable for discs with significant height loss, which also often occurs during the later stages of disc degeneration. When a disc loses height over time, the collagen fibers may adapt into their new structural characteristics and would hence be difficult to stretch significantly at surgery.

Without much prior laboratory testing, Fernstrom ${ }^{14}$ initiated the clinical use of a spherical endoprosthesis consisting of a stainless-steel ball. The diameter of the ball ranged from 10 to $16 \mathrm{~mm}$ for the lumbar disc and from 6 to $10 \mathrm{~mm}$ for the cervical disc. This solid-ball device was meant to serve as a spacer allowing movement of adjacent vertebrae. It did not, for obvious reasons, restore the normal stiffness of the disc. Although the only published long-term clinical study of this device showed reasonably good clinical results in a fairly large group of patients, ${ }^{29}$ its use has been discontinued due to concerns about implant migration and subsidence.

Aware that metals are too stiff for a nucleus device, ${ }^{12,21}$, ${ }^{30,41}$ many researchers have experimented with various elastomers (either preformed or formed in situ) for nucleus replacement. Other than the device invented by Hou, ${ }^{21}$ which was used in a limited number of patients, many of these designs did not meet the basic requirements, such as biocompatibility and mechanical strength, for a clinical trial.

In 1990, Bao and Higham ${ }^{4}$ pioneered research into using hydrogel material for intervertebral nucleus replacement. After having evaluated many different hydrogel materials, they developed a hydrogel nucleus implant designed to contain approximately $70 \%$ water content under physiological loading conditions while also meeting the mechanical requirements. As reported at the 11th annual meeting of the North American Spine Society, these researchers, in collaboration with others were also the first to demonstrate that the hydrogel nucleus implant can mimic the capacity of the nucleus tissue to absorb and release water under cyclic loading conditions. ${ }^{3}$ Biomechanical studies (reported at the aforementioned meeting) have confirmed restoration of disc anatomy and function after implantation of a hydrogel nucleus. A study using a baboon model (unpublished data) has also shown that there is no adverse local or systemic tissue reaction. This nucleus device may well reach the clinical trial stage in the near future.

The nucleus implant in most widespread clinical use is the one developed by RayMedica (Bloomington, MN). In 1988 Ray and Corbin ${ }^{36,37}$ introduced the use of dual disc cylinders for disc nucleus replacement. The threaded outer layer corresponding to the light threads cut into the endplates was a double-woven spiral of flexible, high-tensilestrength polymeric fibers and tissue ingrowth-promoting polyglycolic acid filaments. The flexible and semipermeable cylinders contained a viscous hygroscopic semifluid. Perhaps because of the technical difficulty involved in sealing the aforementioned semifluid into the capsule, this concept was abandoned. In the mid-1990s, Ray, et al., ${ }^{38}$ working for RayMedica, decided to change their approach, using for the core a hydrogel constrained by a flexible but inelastic woven polyethylene-fiber jacket. The shape of the implant was also changed to resemble that of a pillow. The implant position was changed from side-byside in an anterior-posterior direction to a medial-lateral 
direction, mainly to reduce implant extrusion. Due to the size of implant, an incision larger than the normal size for a discectomy procedure has to be made using a posterior approach. To minimize annulus dissection, a flap is made and sutured back after implantation.

RayMedica initiated the clinical trial of this implants in humans in Germany in 1996. ${ }^{36}$ A pilot United States Investigational Device Exemption study was begun in 1998. At the 1st Annual North American Artificial Disc Summit in October 1999, RayMedica reported that 101 patients had received its nucleus implant (unpublished data). Among patients in whom there was no implant extrusion, pain relief was achieved in the majority. However, the company reported that 17 of the first 101 patients did suffer implant extrusion.

More recently, in an effort to minimize implant extrusion and improve artificial disc technology with minimally invasive surgical methods, Advanced BioSurfaces (Minnetonka, MN) has developed a system that features a polymer formulation, a delivery balloon and balloon catheter, and a polymer-injection gun. ${ }^{13}$ The polymer is a polyurethane that can be cured in situ; it has strong mechanical properties comparable to those of other industrially cured medical polyurethanes. The company has conducted extensive in vivo and in vitro studies (unpublished data) to demonstrate the biocompatibility of this polymer during the course of developing this technology for application in knee surgery. In these studies it was shown that monomer-leachable elements are minimal. The balloon is made of a compliant material yet is very robust, which allows significant expansion during polymer injection to fill and conform with the cavity. Due to these characteristics, the device allows the surgeon to distract the disc space under controlled pressure. A biomechanical study in which a human cadaveric disc model was used demonstrated that this device is able to restore both disc height disc modulus.

\section{Disc Prosthesis for Thoracic Disc Replacement}

Thus far, the majority of research-and-development efforts in artificial disc technology have been focused on the lumbar disc, with only a small percentage devoted to the cervical disc. There has been almost no significant research into prostheses for the thoracic disc. One of the undisputed reasons for this disparity is the relative rarity of thoracic disc disease and, therefore, thoracic disc surgery. This type of procedure accounts for less than $1 \%$ of all disc corrective surgeries. ${ }^{43}$ However, because thoracic disc diseases do nevertheless exist and many of them require the same type of surgical treatment as that used for lumbar disc diseases, it may be worthwhile to speculate on the applicability of artificial disc technology in treating thoracic disc disease.

Many different factors, such as structure, anatomy, function, degeneration mechanism, pathology, surgical approach, and complications of thoracic disc surgery, can affect the applicability of artificial disc technology in the treatment of thoracic disc disease.

Structurally, the composition of the discs in all three spinal regions is fairly similar. However, the thoracic spine has several unique characteristics. In this region the spinal cord is relatively small, yet the ratio of spinal cord to spinal canal is larger than that in other regions. Also, the blood supply is more variable in the thoracic spine, with a particularly vulnerable region between T-4 and T-9. The cross-sectional area of thoracic discs is larger than that of cervical discs, but smaller than that of lumbar discs. The variation in disc height does not follow the same trend along the spine, with midthoracic discs being the highest. The shape of thoracic discs is more circular, whereas that of lumbar and cervical discs is more elliptical. ${ }^{34}$ The thoracic spine has a kyphotic curvature, whereas both cervical and lumbar discs are more wedge shaped. ${ }^{34}$

Structurally, it should not be too difficult to transfer artificial disc technology (either total disc prosthesis or disc nucleus prosthesis) from the lumbar region to the thoracic region, apart from the problem of adjusting for differences in size and geometry. The similiarity of shape across the entire length of the thoracic region should entail less emphasis on maintaining normal lordosis than when replacing lower lumbar discs. The greater disc height in the midthoracic region should, in general, make disc replacement easier than in the case of narrower discs. The structural similarity in disc composition should also allow nucleus replacement if annular integrity is not grossly compromised.

The major functions of the thoracic discs are similar to those of cervical and lumbar discs. The compressive load borne by the thoracic discs is lower than that borne by lumbar discs but higher than that borne by cervical discs. Without the support of the rib cage, one would probably expect that thoracic discs would be more susceptible to motion, given their position in the middle of the spinal column. Actually, thoracic disc joints are stiffer, with less range of motion in flexion-extension, than lumbar and cervical joints; this is due to the additional constraining force exerted by costovertebral articulations and the rib cage. The variation in range of motion in lateral bending throughout the thoracic region is not large. The range of motion of axial rotation of lower thoracic discs is similar to that of lumbar discs, whereas that of mid- to upper-thoracic discs is similar to that of cervical discs. ${ }^{47}$

The greater stability of the thoracic disc joint has implications for the applicability of artificial disc technology in treating thoracic disc disease. First, even in degenerative thoracic discs there is less joint instability than in degenerative lumbar and cervical discs. Therefore, in treating thoracic disc pathology, it is not as necessary to reestablish joint stability. Second, the superior stability contributed by nondisc elements such as the rib cage and costovertebral articulation can often better maintain disc joint stability even after disc surgery. For these reasons it is less common to fuse the thoracic disc after discectomy. Because one of the main objectives of artificial disc implantation is to maintain or restore disc function and stability, there will be less need for surgeons to place an artificial disc if they can treat the disease by using current techniques without significantly altering normal mechanical function.

Due to the rarity of thoracic disc disease, the exact mechanism of its degeneration has not been studied as thoroughly as that of lumbar disc degeneration. However, based on structural and functional similarity among discs in all three regions, it is believed that the mechanism of thoracic disc degeneration should be similar to that of lumbar and cervical disc degeneration. The most common result of thoracic disc disease is disc herniation, in which 
the herniated disc material compresses spinal nerves and causes pain. As is the trend in the lumbar region, the majority of disc herniations in the thoracic region occur at the lower levels (T8-T12), probably due to the fact that the lower thoracic discs bear greater stress than the upper thoracic discs. ${ }^{1,2}$ Most herniations occur at either central or centrolateral locations. ${ }^{27,34}$ Although symptomatic herniation at multiple levels has been reported in thoracic discs, it is not as common as it is in lumbar discs.

Discectomy is the surgical procedure most commonly used by surgeons to treat thoracic disc disease. Although autograft bone block placement is sometimes used with discectomy, interbody fusion is not a common procedure for this disease because of the thoracic spine's relative stability. For this reason it would be more difficult to use an artificial total disc because it entails more radical surgery. It may be more effective to replace only the disc nucleus with a prosthesis, if it can be easily implanted using current discectomy methods.

Laminectomy, one of the early surgical treatments for thoracic disc disease, was found to produce disappointing results, ${ }^{15}$ due largely to inadequate decompressive effect of the posterior approach as opposed to direct removal herniated tissue. ${ }^{34}$ Since then, many other surgical techniques have been developed to treat thoracic disc disease, including the following: lateral rhachotomy, first described by Capener; ${ }^{10}$ modified costotransversectomy; ${ }^{22}$ transthoracic surgery; $; 33,35$ transpedicular surgery; ${ }^{32}$ lateral extracavitary surgery; ${ }^{27}$ and transthoracic and costotransversectomy procedures. ${ }^{6}$ These methods involve the same three approaches used for lumbar and cervical discs surgery: posterior, anterior, and lateral. Therefore, it would be feasible to apply any artificial disc or disc nucleus developed for these three approaches to the thoracic disc.

Recently, minimally invasive spinal surgery techniques have also been applied to thoracic discectomy. ${ }^{39,40}$ Videoassisted thoracic surgery, which involves the same anterolateral approach entailed by open thoracotomy, allows surgeons access to the disc spaces, vertebral bodies, paravertebral soft tissues, spinal cord, spinal nerves, and sympathetic chain at all thoracic spine levels. Although the indications for VATS are the same as those for open thoracotomy, VATS offers the advantages of reduced postoperative pain, lower complication rates, and shorter recovery times. If this procedure continues to gain in popularity, artificial disc or nucleus devices that can be implanted by means of endoscopic procedures should have an advantage over those that must be implanted via open surgery.

The last but not the least important factor that can affect the applicability of artificial disc technology in treating thoracic disc disease is the potential risk it may add to currently used surgical procedures. Because artificial disc or disc nucleus devices are designed mainly to improve the long-term results of existing procedures that have known (and relatively low) risk factors and complication rates, it is vital that the use of these prosthetic devices not be allowed to increase significantly these potential liabilities, especially when the clinical benefits have not yet been proven.

Early laminectomy procedures were associated with high rates of paresis, paralysis, or even death. Coupled with poor clinical outcomes, laminectomy was essentially abandoned as a treatment for thoracic disc disease after
1960. The paresis and paralysis rate for anterolateral thoracotomy, one of the most popular procedures for thoracic disc disease, has been reported to be approximately $1 \%$, with no deaths. ${ }^{39}$ The total overall morbidity rate associated with the transthoracic procedure-including pneumonia, atelectasis, pulmonary embolus, postoperative occult compression fracture, deep or superficial wound infections, bowel obstruction, and postoperative seizure-is only approximately $11 \%$. Another common method of thoracic disc surgery is the lateral extracavitary approach, which is associated with approximately the same overall morbidity rate as transthoracic surgery, with no reported deaths. The morbidity rate for other less commonly used approaches, such as transpedicular and costotransversectomy, is also reported to be in the same range. Due to its brief history, the morbidity rate for VATS has been less well documented. Based on the small number of publications, the overall morbidity rate associated with VATS appears to be reasonably low, but only after a learning curve that is steeper than that required for open surgery.

Overall, the morbidity rate associated with current surgical techniques for thoracic disc disease appears to be low, with almost no deaths. ${ }^{15}$ Again, when applying artificial disc technology to the treatment of thoracic disc disease, it is important that the morbidity rate not be increased.

\section{CONCLUSIONS}

Although it is growing more and more clear that artificial disc or disc nucleus implantation will become the treatment of choice for lumbar and cervical disc disease, its application in treating thoracic disc disease may be farther away. The rarity of thoracic disc surgery is the major reason for the lack of research and development activities related to artificial thoracic disc or disc nucleus technology. However, after the safety and efficacy of the technology have been demonstrated in clinical use for the treatment of lumbar and cervical discs, there should be few obstacles to prevent its transfer to the treatment of diseased thoracic discs. Nevertheless, because the thoracic disc joint is more stable, the potential benefits of using an artificial nucleus may not be as great for thoracic as they would be for lumbar or cervical discs. To make artificial disc or disc nucleus implantation more acceptable as a treatment for thoracic disc disease, any added risk and cost associated with this new procedure must be reduced so that the benefit-to-cost and benefit-to-risk ratios are high enough to justify its clinical use.

\section{Disclosure}

Dr. Bao is vice president of research and development at Disc Dynamics, Inc., which is a spin-off of Advanced Biosurfaces, Inc. He was employed by Howmedica, Inc., when he pioneered research into the use of hydrogel material for nucleus prostheses.

\section{References}

1. Alberico AM, Sahni S, Hall JA Jr, et al: High thoracic disc herniation. Neurosurgery 19:449-451, 1986

2. Arce CA, Dohrman GJ: Thoracic disc herniation. Improved diagnosis with computed tomographic scanning and a review of the literature. Surg Neurol 23:356-361, 1985 
3. Bao QB, Higham PA: Hydrogel Iintervertebral Disc Nucleus. US Patent 5,047,055, 10 September 1991

4. Bao QB, Higham PA: Hydrogel Intervertebral Disc Nucleus. US Patent 5,192,326, 9 March 1993

5. Bao QB, McCullen GM, Higham PH, et al: The artificial disc: theory, design and materials. Biomaterials 17:1157-1167, 1996

6. Bohlman HH, Zdeblick TA: Anterior excision of herniated thoracic discs. J Bone Joint Surg (Am) 70:1038-1047, 1988

7. Brock M, Mayer HM, Weigel K (eds): The Artificial Disc. Berlin: Springer-Verlag, 1991

8. Buttner-Janz K, Helisch HJ, Schellnack K, et al: Intervertebral Endoprosthesis. US Patent 4,759,766, 26 July 1988

9. Buttner-Janz K, Schellnack K, Zippel H: Biomechanics of the SB Charité lumbar intervertebral disc endoprosthesis. Int Orthop 13:173-176, 1989

10. Capener N: The evaluation of lateral rachotomy. J Bone Joint Surg (Br) 36:173-179, 1954

11. Enker P, Steffee A, McMillin CR, et al: Artificial disc replacement. Preliminary report with a 3-year minimum follow-up. Spine 18:1061-1070, 1993

12. Fassio B, Ginestie JF: [Discal prosthesis made of silicone: experimental study and 1st clinical cases.] Nouv Presse Med 21: 207, 1978 (Fr)

13. Felt CJ, Bourgeault CA, Baker MW: Articulating Joint Repair. US Patent 5,888,220, 30 March 1999

14. Fernstrom U: Metallic disk prosthesis for lumbar disc rupture. Nord Med 71:160, 1964

15. Fessler RG, Sturgill M: Complications of surgery for thoracic disc disease. Surg Neurol 49:609-618, 1998

16. Goel VK, Nishiyama K, Weinstein JN, et al: Mechanical properties of lumbar spinal motion segments as affected by partial disc removal. Spine 11:1008-1012, 1986

17. Griffith SL, Shelokov AP, Buttner-Janz K, et al: A multicenter retrospective study of the clinical results of the LINK SB Charité intervertebral prosthesis. The initial experience. Spine 9:1842-1949, 1994

18. Hanley EN Jr, Shapiro DE: The development of low-back pain after excision of a lumber disc. J Bone Joint Surg (Am) 71: 719-721, 1989

19. Hedman TP, Kostuik JP, Fernie GR, et al: Design of an intervertebral disc prosthesis. Spine 16 (Suppl 6):S256-S260, 1991

20. Hellier WG, Hedman TP, Kostuik JP: Wear studies for development an intervertebral disc prosthesis. Spine 17 (Suppl 6): S86-S96, 1992

21. Hou TS, Tu KY, Xu YK, et al: Lumbar intervertebral disc prosthesis. An experimental study. Chin Med J 104:381-386, 1991

22. Hulme A: The surgical approach to thoracic intervertebral disc protrusions. J Neurol Neurosurg Psychiatry 23:133-137, 1960

23. Langrana NA, Parsons JR, Lee CK, et al: Materials and design concepts for the intervertebral disc spacer. I. Fiber-reinforced composition design. J Appl Biomater 5:125-132, 1995

24. Lee CK, Langrana NA: Lumbosacral fusion. A biomechanical study. Spine 9:574-581, 1984

25. Lee CK, Langrana NA, Alexander H, et al: Functional and Biocompatible Intervertebral Disc Spacer. US Patent 4,911,718, 27 March 1990

26. Lemaire JP, Skalli W, Lavaste F, et al: Intervertebral disc prosthesis-results and prospects for the year 2000. Clin Orthop (337):64-76, 1997

27. Maiman DJ, Larson SJ, Luck E, et al: Lateral extracavitary approach to the spine for thoracic disc herniation: report of 23 cases. Neurosurgery 14:178-182, 1984

28. Marnay T: Prosthesis for Intervertebral Discs and Instruments for Implanting It. US Patent 5,314,477, 24 May, 1994
29. McKenzie AH: Ferstrom intervertebral disc arthroplasty: a long-term evaluation. Orthop Int 3:313-324, 1995

30. Nachemson A: Some mechanical properties of the lumbar intervertebral disc. Bull Hosp Joint Dis 23:130-132, 1962

31. Parson JR, Lee CK, Langrana NA, et al: Functional and Biocompatible Intervertebral Disc Spacer Containing Elastomeric Material of Varying Hardness. US Patent 5,171,281, 5 December 1992

32. Patterson RH Jr, Arbit E: A surgical approach through the pedicle to protruded thoracic discs. J Neurosurg 48:768-772, 1978

33. Perot PL Jr, Munro DD: Transthoracic removal of midline thoracic disc protusions causing spinal cord compression. J Neurosurg 31:452-458, 1969

34. Pooni, JS, Hukins DWL, Harris, PF, et al: Comparison of the structure of human intervertebral discs in the cervical, thoracic and lumbar regions of the spine. Surg Radiol Anat 8:175-182, 1986

35. Ransohoff J, Spencer F, Siew F, et al: Transthoracic removal of thoracic discs. Reports of three cases. J Neurosurg 31: 459-461, 1969

36. Ray CD: Lumbar interbody threaded prostheses. Flexible, for an artificial disc and rigid, for a fusion, in Brock M, Mayer HM, Weigel K (eds): The Artificial Disc. Berlin: Springer-Verlag, 1991, pp 53-67

37. Ray CD, Corbin TP: Prosthetic Disc and Method of Implanting. US Patent 4,772,287, 20 September 1988

38. Ray CD, Dickhudt EA, Ledoux PJ, et al: Prosthetic Spinal Disc Nucleus. US Patent 5,674,295, 7 October 1997

39. Regan JJ, Mack MJ, Picetti GD: A technical report on videoassisted thoracoscopy in thoracic spine surgery. Spine 20: 831-837, 1995

40. Rosenthal D, Rosenthal R, da Simone A: Removal of a protruded thoracic disc using microsurgical endoscopy. A new technique. Spine 19:1087-1091, 1994

41. Roy-Camille R, Saillant G, Lavaste F: Experimental study of lumbar disc replacement. Rev Chir Orthop (Suppl 2) 64: 106-107, 1978

42. Steffee AD: The Steffee artificial disc, in Weinstein JN (ed): Clinical Efficacy and Outcome in the Diagnosis and Treatment of Low-back Pain. New York: Raven Press, 1992, pp 245-258

43. Stillerman CB, Weiss MH: Management of thoracic disc disease. Clin Neurosurg 38:325-352, 1991

44. Urbaniak JR, Bright DS, Hopkins JE: Replacement of intervertebral discs in Chimpanzees by silicone-Dacron implants: a preliminary report. J Biomed Mater Res Symp 4:165-186, 1973

45. van Steenbrugghe MH: Improvements in Joint Prosthesis. French Patent 1,122,634, 28 May 1956

46. Weinstein JN (ed): Clinical Efficacy and Outcome in the Diagnosis and Treatment of Low Back Pain. New York: Raven Press, 1991

47. White AA, Panjabi MM (eds): Clinical Biomechanics of the Spine, ed 2. Philadelphia: Lippincott-Williams \& Wilkins, 1990

48. Yong-Hing K, Kirkaldy-Willis WH: The three-joint complex, in Weinstein JN, Wiesel SW (eds): The Lumbar Spine. Philadelphia: WB Saunders, 1990, pp 80-87

49. Zippel H: "Charité modular:" concept, experience and results, in Brock M, Mayer HM, Weigel K (eds):The Artificial Disc. Berlin: Springer-Verlag, 1991, pp 69-77

Manuscript received August 14, 2000.

Accepted in final form September 13, 2000.

Address reprint requests to: Qi-Bin Bao, Ph.D., Disc Dynamics, Inc., 5909 Baker Road, Suite 550, Minnetonka, Minnesota 55345. email: cbao@advbiosurf.com. 